\title{
The secretome of bone marrow mesenchymal stem cells-conditioned media varies with time and drives a distinct effect on mature neurons and glial cells (primary cultures)
}

\author{
C. A. Ribeiro ${ }^{1 \#}$, A. J. Salgado ${ }^{1 \#}$, J. S. Fraga ${ }^{1}$, N. A. Silva ${ }^{1,2,3}$, R. L. Reis ${ }^{2,3}$ and N. Sousa ${ }^{1 *}$ \\ ${ }^{1}$ Life and Health Sciences Research Institute (ICVS), School of Health Sciences, University of Minho, Braga, Portugal \\ ${ }^{2} 3$ Bs Research Group - Biomaterials, Biodegradables and Biomimetics, University of Minho, Headquarters of the European Institute of \\ Excellence on Tissue Engineering and Regenerative Medicine, AvePark, Taipas, Guimarães, Portugal \\ ${ }^{3}$ Institute for Biotechnology and Bioengeneering, PT Government-Associated Laboratory, Guimarães, Portugal
}

\begin{abstract}
Transplantation of bone marrow mesenchymal stem cells (BM-MSCs) has been shown to ameliorate the injured central nervous system (CNS). Although these effects were initially attributed to the putative differentiation of MSCs towards the neural lineage, it is now known that most of them are mediated by the secretome. Up to now most in vitro reports have dealt with the effects of the secretome on neural stem cells and their differentiation. Consequently, there is a lack of information regarding the role of the secretome on the viability and survival of pre-existent matured differentiated cell populations. Moreover, it is also not known how the time points of conditioned media (CM) collection affect such parameters. In the present study, primary cultures of hippocampal neurons and glial cells were incubated with CM obtained from MSCs. To determine how the temporal profiles of CM collection impact on post-natal neurons and glial cells, we collected MSCs CM at 24, 48, 72 and $96 \mathrm{~h}$ of conditioning. MTS test revealed that for the hippocampal cultures the incubation with CM increased cell viability for all time points, with significant increases in the percentage of neurons in culture incubated with CM $24 \mathrm{~h}$. For glial cells only the later time point of CM collection $(96 \mathrm{~h})$ increased cell viability. Fluorescence microscopy observations also revealed that CM $48 \mathrm{~h}$ and $72 \mathrm{~h}$ increased astrocytes percentages, while CM $24 \mathrm{~h}$ decreased microglial cell and oligodendrocytes values. These results revealed that post-natal neuronal and glial cells respond differently to MSCs CM; moreover, there are specific temporal variations in the composition of the CM of MSCs collected at different time points that trigger different effects on mature neurons and the distinct glial cell populations (astrocytes, oligodendrocytes and microglial cells). Copyright $\odot$ 2011 John Wiley \& Sons, Ltd.
\end{abstract}

Received 23 June 2010; Accepted 29 July 2010

Keywords bone marrow; mesenchymal stem cells; temporal profile; neurons; glia; secretome

\section{Introduction}

Mounting evidence indicates the possible use of Mesenchymal Stem Cells (MSCs) as therapeutical agents

\footnotetext{
*Correspondence to: N. Sousa, Life and Health Sciences Research Institute (ICVS), School of Health Sciences, University of Minho, Campus de Gualtar, 4710-057 Braga, Portugal. E-mail:njcsousa@ecsaude.uminho.pt

\# These authors contributed equally to the work.
}

for different CNS-related problems. Most studies have focused on MSCs' impact on phenomena such as neural cell proliferation and differentiation. For instance, Munoz et al. (2005) revealed that the implantation of human MSCs in the mousee dentate gyrus (DG) stimulated proliferation, migration and differentiation of the endogenous neural stem cells populations. Other authors have also revealed that the transplantation of MSCs into different animal injury/disease models, such as stroke (Li et al., 2005; Li and Chopp, 2009), spinal cord injury (SCI) (Deng 
et al., 2006; Urdziková et al., 2006) or Parkinson's Disease (PD) (Chao et al., 2009; Park et al., 2008; Shintani et al., 2007) led to a significant reduction in the volume of damaged tissues, increased cognitive capabilities upon injury or a significant improvement in motor skills. While these effects can be ascribed to the proliferation/differentiation of local neural stem cell (NSC) populations, there is also a strong possibility that they are related to the trophic influence on the survival and protection of mature resident glial and neuronal cell populations. Reports such as those presented by Bai et al. (2007), Crigler et al. (2006) and Wislet-Gendebien et al. (2004) clearly indicate that the secretome of MSCs possesses regulatory factors able to stimulate neuronal, oligodendrogenic and astrocytic survival/protection and differentiation. Nevertheless, although there has been an increasing body of literature dealing with effects of the secretome of MSCs in the CNS, there are still some missing gaps within the field. For instance, few reports deal with the effects of the secretome of MSCs on mature cultures of postnatal neurons and glial cells, as most in vitro studies have been performed with NSCs. Moreover, as the secretome of MSCs might change with time, it is also relevant to characterize the temporal profile of conditioned media (CM) collection in terms of cell viability, survival and cell densities, as subtle changes in the CM might stimulate some cell populations while inhibiting others. Therefore, the objectives of the present study were to evaluate the effects of MSCs conditioned media and the temporal profile of its collection on the viability and cell densities of post-natal cultures of hippocampal neurons and glial cells. The results revealed that the time point of MSCs CM collection distinctly affects neurons, astrocytes, oligodendrocytes and microglial cells.

\section{Materials and methods}

Bone marrow MSCs (BM-MSCs) were acquired from Lonza (Basel, Switzerland). Upon arrival they were thawed and expanded according to the company guidelines. Hippocampal neuron cultures were prepared from P4 Wistar rats (Lu et al., 2005). Briefly, and upon dissection, the hippocampi were submitted to trypsin-based enzymatic digestion followed by mechanical dissociation. Isolated cells were then plated on coverslips previously coated with poly-D-lysine (Sigma, USA) at a density of 40000 cells $/ \mathrm{cm}^{2}$. Cortical glial cells were isolated from P4 newborn Wistar rats (Salgado et al., 2009). Upon dissection, the cortices were enzymatically digested $(30 \mathrm{mg} / \mathrm{ml}$ DNase, $0.25 \%$ trypsin; both from Sigma), followed by strong mechanical dissociation. Glial cells were then obtained by centrifuging the resulting cell suspension at $800 \mathrm{rpm}$ for $2 \mathrm{~min}$ and were plated out on coverslips previously coated with poly-D-lysine at a density of 40000 cells $/ \mathrm{cm}^{2}$. CM were collected from P5 MSCs. For this purpose, the cells were plated out at a density of 4000 cells $/ \mathrm{cm}^{2}$ and allowed to grow for 3 days. Following this, the culture medium was renewed and CM collected 24, 48, 72 and $96 \mathrm{~h}$ thereafter (cell culture medium was not renewed or added during this time period). Upon collection, the CMs were frozen and later thawed on the day of the experiments. For CM collection, standard MSCs culture medium was used for experiments with glial cells, while for experiments with hippocampal neurons, Neurobasal-A medium (Gibco, USA) supplemented with kanamycin ( $0.1 \mathrm{mg} / \mathrm{ml}$; Gibco) was the chosen medium.

Experiments with glial cells and hippocampal neurons were performed as follows.

Hippocampal neurons. Upon isolation, hippocampal neurons were plated out at the densities referred to above and incubated from $T_{0}$ with the previously collected $\mathrm{CM}$ ( $n=3$ /CM time point) for 7 days (with half of the volume of CM being renewed at day 4 of culture), after which cell densities, viability and proliferation were assessed (see below). Besides kanamycin and glutamax, no further supplements were added to the MSCs CM. Control cultures were kept in neurobasal medium supplemented with kanamycin and glutamax.

Glial cells. Upon isolation, glial cells were allowed to grow for 5 days. On day 5 the culture medium was switched to $\mathrm{CM}$ ( $n=3 / \mathrm{CM}$ time point); after incubation for further 9 days, cell densities and viability were assessed (see below). Control cultures were kept in $\alpha$-MEM supplemented with $1 \%$ antibiotics/antimycotics (Sigma). The different time periods of CM incubation for neurons and glial cells were mainly related to their maturation stage. For hippocampal neurons, the maturation stage is 7 days, while for cortical glial cells it is 14 days (Lu et al., 2005; Salgado et al., 2009).

Cell viability was assessed by the MTS test. Cell culture coverslips $(n=3)$ were placed in culture medium containing (3-(4,5-dimethylthiazol-2-yl)-5-(3carboxymethoxyphenyl)-2(4-sulfophenyl)-2H tetrazolium) (MTS) in a 5:1 ratio and incubated in a humidified atmosphere at $37^{\circ} \mathrm{C}$ and $5 \% \mathrm{CO}_{2}$. After $3 \mathrm{~h}$ of incubation, $100 \mu \mathrm{l}$ solution from each sample were transferred to 96 -well plates and the optical density was determined at $490 \mathrm{~nm}(n=3 / \mathrm{CM}$ time point \pm SD).

Immunocytochemistry was performed according to our previously described procedures (Salgado et al., 2010). The following primary and secondary antibodies were used: mouse anti-rat microtubule-associated protein 2 (MAP-2; Sigma, USA) to detect mature hippocampal neurons; rabbit anti-rat glial fibrillary acid protein (GFAP; Dako, Denmark) for astrocytes; mouse anti-CD11b (BD Biosciences, Pharmigen, USA) for microglial cells; mouse anti-O4 (R\&D Systems, USA) for oligodendrocytes; Alexa Fluor 594 goat anti-mouse immunoglobulin G (IgG) and Alexa Fluor 488 rabbit anti-rat IgG. Nuclei were counterstained with DAPI (Molecular Probes). Primary antibody was omitted to produce negative controls. Samples were observed under a Olympus BX-61 fluorescence microscope (Olympus, Germany).

Cell counts were performed by using Cell-P software (Olympus, Germany). For this purpose, three cover slips per condition and three representative fields were chosen 
and analysed. In order to normalize the data between the different sets of cell cultures, the results are presented in percentages of cells. This was calculated by counting the cells positive for the above-referred markers and dividing this value by the total number of cells/field (DAPI-positive cells). Statistical evaluation was performed using oneway ANOVA to assess the statistical differences between different groups. Statistical significance was defined as $p<0.05$ for a $95 \%$ confidence interval.

\section{Results and discussion}

In the present report we aimed to determine how $\mathrm{CM}$ from MSCs collected at different time points affected the metabolic viability and cell densities in primary cultures of post-natal (P4) hippocampal neurons and cortical glial cells. As can be observed in Figure 1A, the incubation of hippocampal neuronal cultures with MSCs $\mathrm{CM}$ leads to an increase of the metabolic viability; values from all time points were above control cultures (CM $24 \mathrm{~h}$ and CM $72 \mathrm{~h}, p<0.05)$. Analysis of differentiated neurons (by observing MAP-2 immunostained coverslips) revealed that $\mathrm{CM} 24 \mathrm{~h}$ triggers the most striking effect on neuronal densities (Figure 1B, C): with this CM, approximately $20 \%(p<0.05)$ of MAP-2-positive cells could be observed, which corresponds to four times more than those presented in the control condition $(p<0.05)$. It should be noted that the low values obtained by the control were expected, as cultures were kept without the B27 supplement. In primary cultures of postnatal cortical glial cells, the results for the metabolic viability upon incubation with MSCs CM were variable. As can be observed in Figure 2A, the two initial CM time points had lower levels of metabolic viability when compared to control cultures $(p<0.05)$. This effect was then inverted with CM 96 h, disclosing a small but statistically significant increase in glial cell viability $(p<0.05)$ when compared to the control. Immunostaining for GFAP (astrocytes; Figure 2B, E, H), CD11b (microglial cells; Figure 2C, F, I) and $\mathrm{O} 4$ (oligodendrocytes; Figure 2D, G, $\mathrm{J})$ revealed distinct effects at the subpopulation level. The most relevant effects for astrocytes were achieved with MSCs CM $48 \mathrm{~h}$ and CM $72 \mathrm{~h}(p<0.05)$. On the other hand, we observed reduced microglial cells densities whenever cultures were incubated with MSCs CM $24 \mathrm{~h}$. Finally, oligodendrocytes densities seem to be negatively affected by the initial MSCs CM (24 h and $48 \mathrm{~h}$ ). While the results obtained with microglial cells were expected, as it is known the immunomodulatory phenotype of MSCs on inflammatory cells, including microglial cells (Kim et al., 2009; Ohtaki et al., 2008; Rossignol et al., 2009; Zhou et al., 2009), the results obtained for astrocytes and oligodendrocytes were quite surprising. Previous
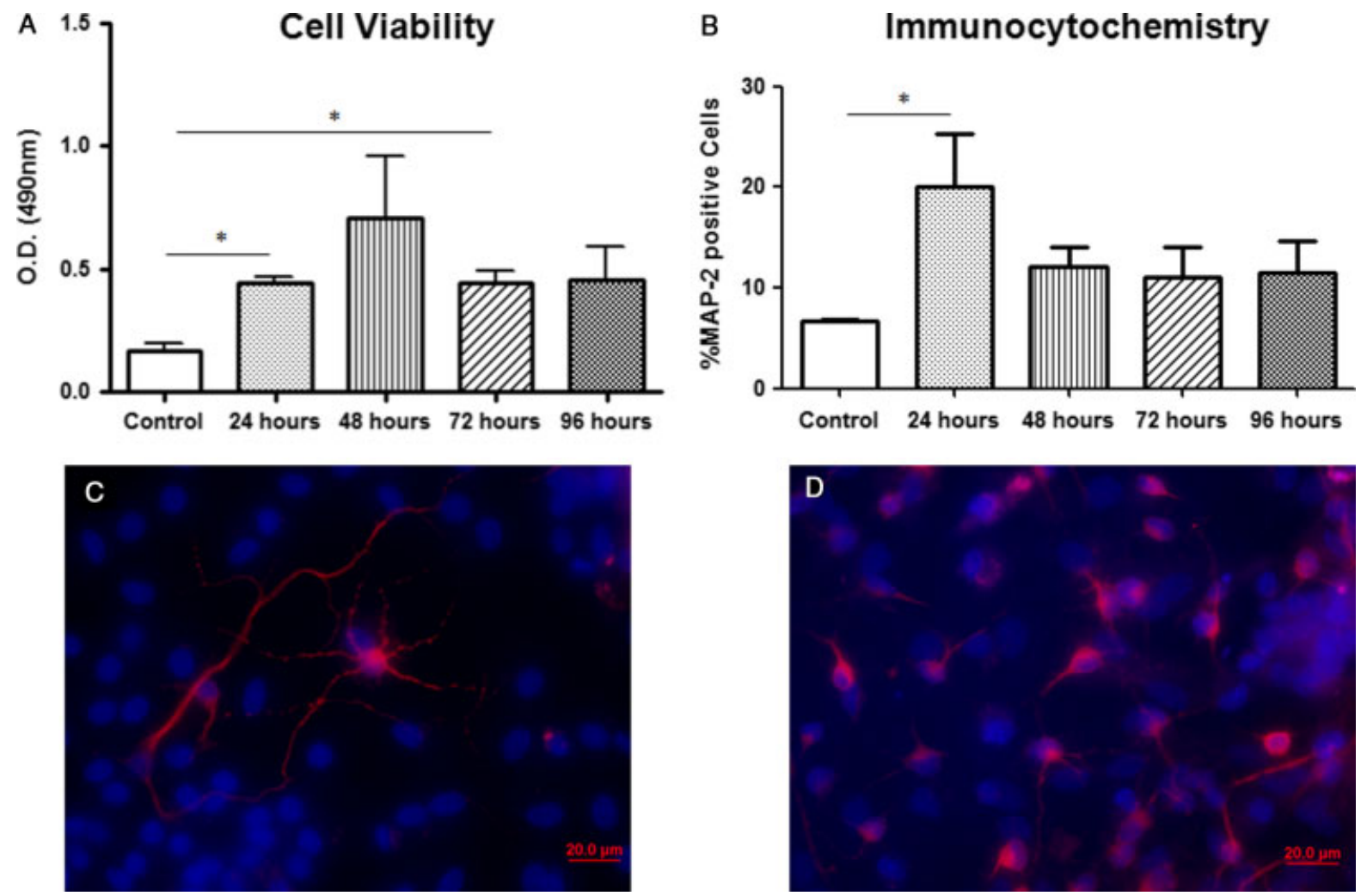

Figure 1. Hippocampal neurons incubated with MSCs CM. MTS test (A) revealed that all the CM time points induced higher rates of metabolic viability when compared to control cultures (CM $24 \mathrm{~h}$ and CM $72 \mathrm{~h}$ were statistically significantly different from the control; one-way ANOVA, $n=3$, mean $\pm S D, p<0.05$ ). For immunocytochemistry (B-D), MAP-2 was used to label hippocampal neurons in cultures. Cell counts revealed that CM $24 \mathrm{~h}$ incubated cultures (B, D) had a higher overall percentage of MAP-2-positive neurons (statistically significantly different from the control; one-way ANOVA, $n=3$, mean \pm SD, $p<0.05$ ) when compared to controls $(\mathrm{B}, \mathrm{C})$ 
A

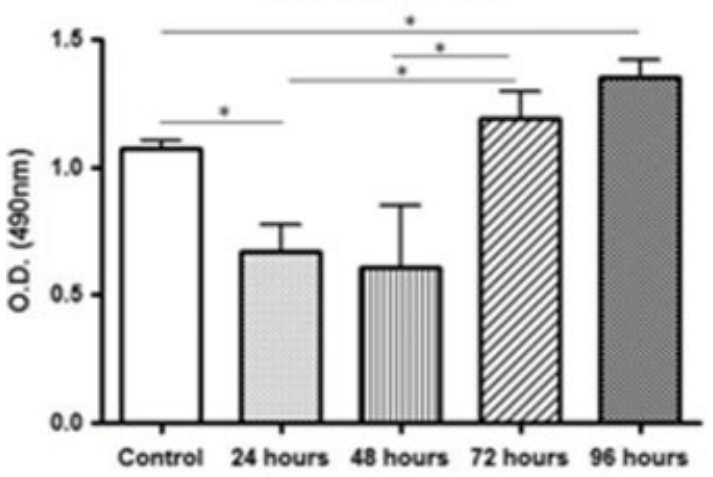

C

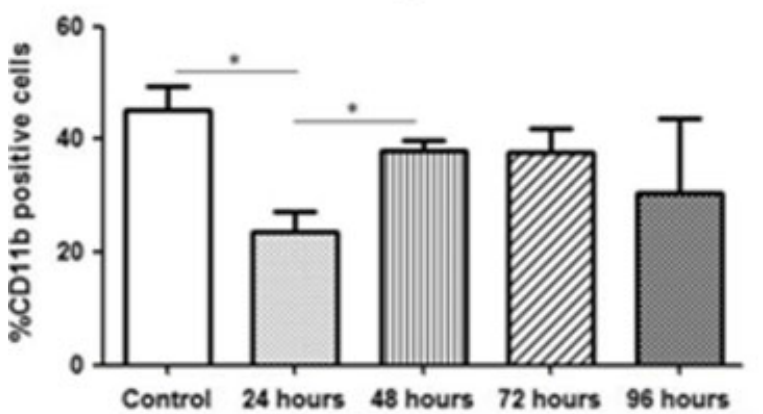

B

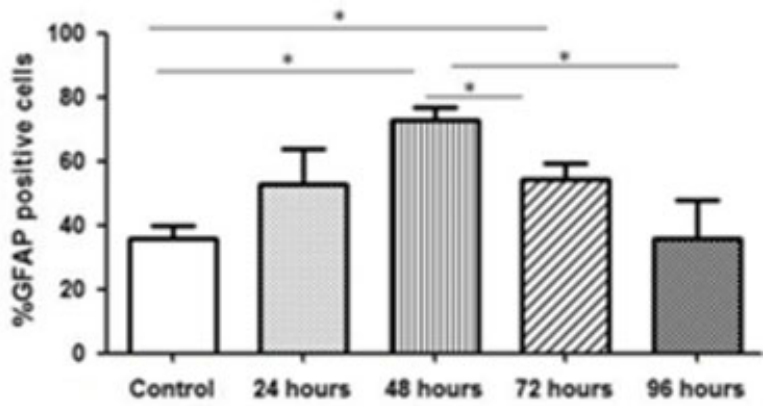

D

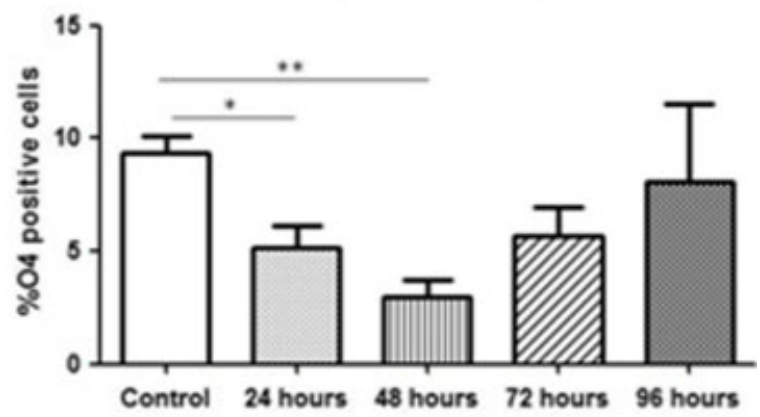

Astrocytes

Oligodendrocytes

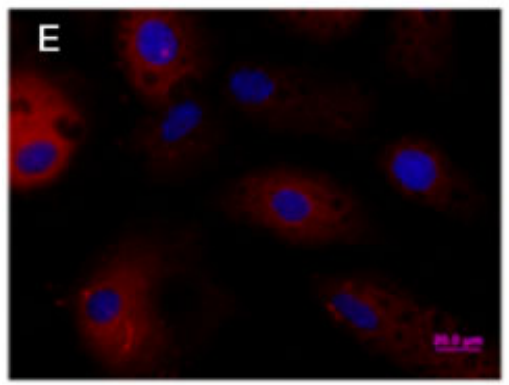

H

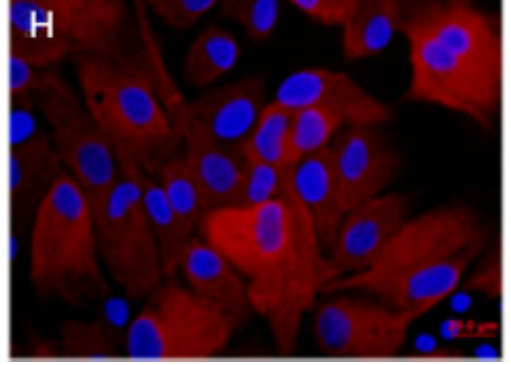

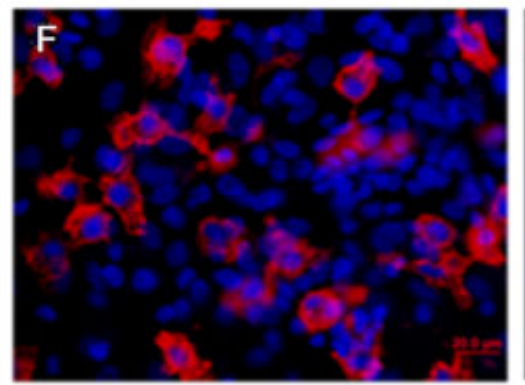

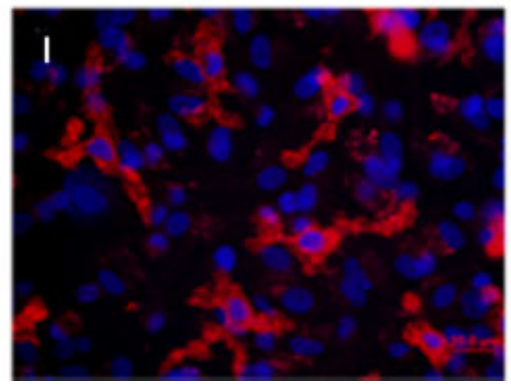

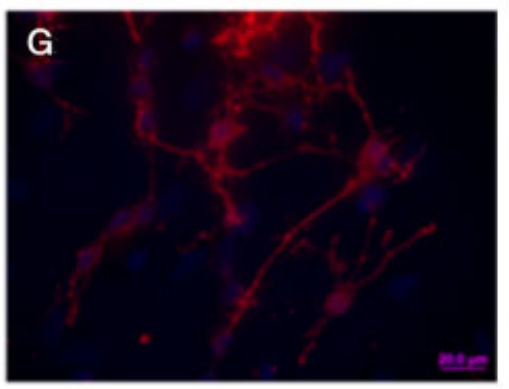

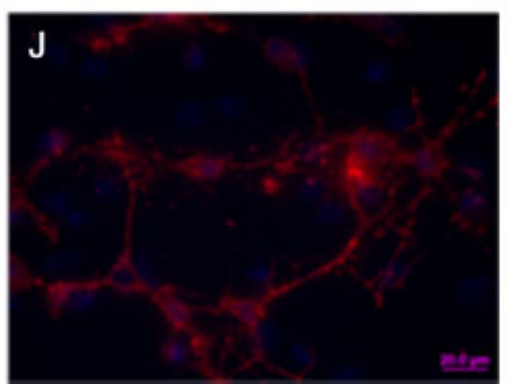

Figure 2. Glial cell incubation with MSCs CM. Cell metabolic viability (A) seemed to be affected for earlier CM time points (24 h and $48 \mathrm{~h}$; CM $24 \mathrm{~h}$ statistically significantly different from the control; one-way ANOVA, $n=3$, mean \pm SD, $p<0.05$ ). With increasing CM time point collections, namely CM $96 \mathrm{~h}$ (statistically significantly different from the control; one-way ANOVA, $n=3$, mean \pm $\mathrm{SD}, p<0.05$ ) these effects were inverted. Immunocytochemistry for GFAP (astrocytes; B, E, H), CD11b (microglial cells; C, F, I) and oligodendrocytes (O4; D, G, J) revealed that the incubation of glial cell cultures with CM $48 \mathrm{~h}$ and CM $72 \mathrm{~h}$ increased (statistically significantly different from the control, one-way ANOVA, $n=3$, mean $\pm S D, p<0.05$ ) the percentage of astrocytes. On the other hand, CM $24 \mathrm{~h}$ induced a decrease in the percentages of microglial cell and oligodendrocytes (statistically significantly different from the control; one-way ANOVA, $n=3$, mean $\pm \mathrm{SD}, p<0.05$ )

reports (Bai et al., 2007; Riviera et al., 2006; WisletGendebien et al., 2004) revealed that NSCs differentiate into oligodendrocytes to the detriment of astrocytes when incubated with MSCs CM or maintained in co-cultures using transwell systems. However, herein we demonstrate that these distinct effects are dependent on the time of $\mathrm{CM}$ collection; indeed, whereas collection of CM at earlier time points induce higher densites of astrocytes, later time points promote a higher survival rate of oligodendrocytes. The latter observation fits previous studies reporting that 
oligodendrogenic differentiation was only achieved with longer periods of MSC conditioning (Bai et al., 2007). Therefore, these results show that the temporal profile of the CM secretome determines its effects on mature and differentiated cells.

\section{Acknowledgements}

We would like to acknowledge Fundação Calouste de Gulbenkian for funds attributed to A. J. Salgado.

\section{References}

Bai L, Caplan A, Lennon D, et al. 2007; Human mesenchymal stem cells signals regulate neural stem cell fate. Neurochem Res 32(2): 353-362.

Chao YX, He BP, Tay SS. 2009; Mesenchymal stem cell transplantation attenuates blood-brain barrier damage and neuroinflammation and protects dopaminergic neurons against MPTP toxicity in the substantia nigra in a model of Parkinson's disease. $J$ Neuroimmunol 216(1-2): 39-50.

Crigler L, Robey RC, Asawachaicharn A et al. 2006; Human mesenchymal stem cell subpopulations express a variety of neuro-regulatory molecules and promote neuronal cell survival and neuritogenesis. Exp Neurol 198(1): 54-64.

Deng YB， Liu XG， Liu ZG, et al. 2006; Implantation of BM mesenchymal stem cells into injured spinal cord elicits de novo neurogenesis and functional recovery: evidence from a study in rhesus monkeys. Cytotherapy 8(3): 210-214.

Kim YJ, Park HJ, Lee G, et al. 2009; Neuroprotective effects of human mesenchymal stem cells on dopaminergic neurons through anti-inflammatory action. Glia 57(1): 13-23.

Lu J, Wu Y, Sousa N, et al. 2005 SMAD pathway mediation of BDNF and TGF $\beta 2$ regulation of proliferation and differentiation of hippocampal granule neurons. Development, 132(14): 3231-3242.

Li Y, Chen J, Zhang CL, et al. 2005; Gliosis and brain remodeling after treatment of stroke in rats with marrow stromal cells. Glia 49(3): 407-417.
Li Y, Chopp M. 2009; Marrow stromal cell transplantation in stroke and traumatic brain injury, Neurosci Lett 456(3): $120-123$.

Munoz JR, Stoutenger BR, Robinson AP et al. 2005; Human stem/progenitor cells from bone marrow promote neurogenesis of endogenous neural stem cells in the hippocampus of mice. Proc Natl Acad Sci USA 102(50): 18171-181716.

Ohtaki H, Ylostalo JH, Foraker JE, et al. 2008; Stem/progenitor cells from bone marrow decrease neuronal death in global ischemia by modulation of inflammatory/immune responses. Proc Natl Acad Sci USA 105(38): 14638-14643.

Park HJ, Lee PH, Bang OY, et al. 2008, Mesenchymal stem cells therapy exerts neuroprotection in a progressive animal model of Parkinson's disease. J Neurochem 107(1): 141-151.

Rivera FJ, Couillard-Despres S, Pedre X, et al. 2006; Mesenchymal stem cells instruct oligodendrogenic fate decision on adult neural stem cells. Stem Cells 24(10): 2209-2219.

Rossignol J, Boyer C, Thinard R, et al. 2009 ; Mesenchymal stem cells induce a weak immune response in the rat striatum after allo- or xenotransplantation. J Cell Mol Med 13(8B): 2547-2558.

Salgado AJ, Sousa RA, Fraga JS, et al. 2009 Effects of starch/polycaprolactone-based blends for spinal cord injury regeneration in neurons/glial cells viability and proliferation. J Bioact Comp Polym 24(3): $235-248$
Salgado AJ, Fraga JS, Mesquita AR, et al. 2010; Role of human umbilical cord mesenchymal progenitors conditioned media in neuronal/glial cell densities, viability and proliferation. Stem Cells Dev 19(7): 1067-1074.

Shintani A, Nakao N, Kakishita K, et al. 2007; Protection of dopamine neurons by bone marrow stromal cells. Brain Res 1186: 48-55.

Urdzíková L， Jendelová $\mathrm{P}$ ， Glogarová $\mathrm{K}$ et al. 2006; Transplantation of bone marrow stem cells as well as mobilization by granulocyte-colony stimulating factor promotes recovery after spinal cord injury in rats. $J$ Neurotrauma 23(9): 1379-1391.

Wislet-Gendebien S, Bruyère F, Hans G, et al. 2004; Nestin-positive mesenchymal stem cells favour the astroglial lineage in neural progenitors and stem cells by releasing active BMP4. BMC Neurosci 5: 33.

Zhou C, Zhang C, Chi S, et al. 2009; Effects of human marrow stromal cells on activation of microglial cells and production of inflammatory factors induced by lipopolysaccharide. Brain Res 1269: $23-30$. 